\title{
Sustainability Strategies for Strengthening Agricultural Systems in Quimiag Parish, Riobamba Canton
}

\section{Estrategias de Sostenibilidad para el Fortalecimiento de Sistemas Agropecuarios en la Parroquia Quimiag, Cantón Riobamba}

I International Seminar of Livestock and Agroindustrial Production ESPOCH 2020

Corresponding Author:

M. Moscoso Gómez

marcelo.moscoso@espoch.edu.ec

Published: 2 September 2021

Production and Hosting by

Knowledge $E$

(c) M. Moscoso Gómez et al. This article is distributed under the terms of the Creative Commons Attribution License, which permits unrestricted use and redistribution provided that the original author and source are credited.
G OPEN ACCESS

\section{Moscoso Gómez ${ }^{1}$, M. Arévalo Lara ${ }^{2}$, D. Loroña Alarcón¹, and D. Campov- erde Santos ${ }^{1}$}

${ }^{1}$ Carrera de Zootecnia, Facultad de Ciencias Pecuarias, Escuela Superior Politécnica de Chimborazo, Riobamba, Ecuador

${ }^{2}$ Particular, Riobamba, Ecuador

\section{Abstract}

The Quimiag parish in Riobamba canton is purely agricultural. For this reason, this study aimed to develop sustainability strategies to strengthen its agricultural systems. 24 communities were involved in the research and 312 Agricultural Productive Units (UPAs) were surveyed. Basic information was collected and then a proposal was developed using the logical framework matrix methodology and focusing on 3 sustainability components: social, productive and economical. In terms of the social component, it was observed that producers were on average 46 years old and $65 \%$ of producers had a primary education. In terms of the production component, we found that maize, beans and potato are the main agricultural products, while dairy cattle, cuyes and pigs are the main livestock in the area. Capacity building, production technology, production credit management and the generation of partnerships with state institutions and private companies are the main sustainability strategies proposed for the Quimiag parish, and adopting these would lead to improvements in profitability rates.

Keywords: sustainability, agricultural systems, economic indicators, Quimiag.

\section{Resumen}

La parroquia Quimiag perteneciente al cantón Riobamba, es netamente agropecuaria, por este motivo el presente estudio tiene por objetivo crear estrategias de sostenibilidad para fortalecer los sistemas agropecuarios de sus productores. Para realizar la investigación se contó con la participación de 24 comunidades y de ellas se muestreo a 312 Unidades Productivas Agropecuarias (UPAs), a las cuales se les aplicó una encuesta que permitió levanter información base para posteriormente elaborar la propuesta utilizando la metodología de matriz de marco lógico, y la cual está enfocada en 3 componentes de sostenibilidad que son: social, productivo y económico. En el componente social se obtuvo que los productores tienen una edad promedio de 46 años y el $65 \%$ de los productores tienen instrucción primaria. En el componente productivo se obtuvo que el maíz, fréjol y papa son los principales productos agrícolas, mientras que el ganado lechero, cuyes y cerdos son los principales rubros pecuarios de la zona. EI fortalecimiento de capacidades, tecnificación de la producción, gestión de créditos productivos y generación de alianzas con instituciones del estado y empresas privadas, son las principales estrategias para la sostenibilidad de la producción de la parroquia Quimiag con los cuales se mejoraría los índices de rentabilidad. 
Palabras Clave: sostenibilidad, sistemas agropecuarios, indicadores económicos, quimiag.

\section{Introducción}

Quimiag es una parroquia rural del cantón Riobamba, provincia de Chimborazo, que cuenta con una superficie de 13949, 63 ha, de las cuales se ha establecido que la mayor parte $(36,6 \%)$ del uso actual del suelo está destinado a la producción de pastos, utilizado este insumo como alimento para el ganado bovino de leche, destacándose esta parroquia por la producción agropecuaria, siendo las lecheras y queseras las mayores iniciativas existentes. La producción agrícola de Quimiag se basa en los rubros: maíz que representa el 31,9\%, papa el $44,5 \%$ habas el $23,6 \%$, siendo el autoconsumo el principal destino de la producción y, en cuanto a la producción pecuaria se basa en el ganado bovino de leche (4988 animales), ganado porcino (2035 animales) y cuyes (19.668 animales), siendo el destino de estos rubros para autoconsumo y venta [1, 2].

El desarrollo sostenible es una preocupación que ha existido en las últimas décadas, en vista que hay un creciente deterioro de los recursos naturales por un proceso extractivista que ha evidenciado la pérdida del vínculo existente entre el desarrollo económico y social y sus efectos más o menos inmediatos sobre el medio natural, generando un fuerte impacto en el cambio climático $[3,4]$.

Los conceptos de desarrollo sostenible y bienestar social están muy conectados, si no existe un desarrollo sostenible favorable no se logra mejorar el bienestar social [5] ya que su accionar influye en las necesidades del presente sin perjudicar las posibles acciones que se consideren en un futuro, si una economía es decreciente sus factores son afectados de manera proporcional de acuerdo con el número de habitantes de la población, ocasionando un decrecimiento del producto per cápita [6-8], además, el desarrollo sostenible implica un proceso de búsqueda de los recursos que aseguren el beneficio individual y social [9], el desarrollo tiene una connotación cualitativa, supone una forma de organización social y una estructuración del aparato productivo que está vinculado con las innovaciones tecnológicas apropiadas [10].

Todo el proceso efectuado por la población en la búsqueda de estrategias para permitan mejorar sus capacidades y elegir oportunamente las mejores decisiones en conjunto son parte del desarrollo humano, incluyendo el perfeccionamiento de sus habilidades para un mejor control de los factores que podrían afectar sus necesidades básicas en sus vidas [11, 12], principalmente impedir que sus limitaciones como el acceso a la educación básica [13], el acceso al agua potable, el derecho a la salud, sean exterminados. Todo este accionar de la población al mejorar sus habilidades y su uso adecuado de los recursos sin afectar el medio ambiente $[14,15]$ preservando su riqueza natural, proporcionará mejores oportunidades, coadyuvando al diseño e implementación de políticas y programas, evitando afectar sus condiciones de vida [16-18].

Para llegar a un desarrollo sostenible, las políticas y acciones de crecimiento económico deben respetar el medio ambiente y ser socialmente equitativas para 
alcanzar un crecimiento económico, siendo estas las 3 dimensiones en las que hay que trabajar, siendo indisociables [19]. A pesar de esto existe la necesidad de una interpretación integrada de estas tres dimensiones, esta postura se ve fortalecida por el enfoque de La Comisión Mundial sobre Medio Ambiente y Desarrollo, que da la razón que estas 3 dimensiones están interrelacionadas y en conjunto deben integrarse para la toma de decisiones sobre el desarrollo presente y futuro [20].

Por lo mencionado, el presente estudio se centró en levantar información de los sistemas de producción de 24 comunidades de la parroquia Quimiag [21], misma que sirvió de insumo para realizar una propuesta de desarrollo sostenible que aborda las 3 dimensiones antes mencionadas. Además, la propuesta se realizó mediante el uso de la matriz de marco lógico que es una herramienta utilizada para apoyar el proceso de análisis, diseño, ejecución y evaluación de proyectos, que orienta al cumplimiento de objetivos de manera participativa y articulada entre diferentes actores [22].

La herramienta de Marco Lógico está orientado al diseño y ejecución de proyectos, además proporciona las técnicas para la evaluación del cumplimiento de las metas trazadas en las propuestas de mejora, es por esto que las organizaciones apoyados en esta herramienta solicitan que estos indicadores sean presentados de una manera coherente para solicitar el apoyo de varias organizaciones [23].

\section{Materiales y Métodos}

El estudio se realizó en 24 comunidades de la parroquia Quimiag, perteneciente al cantón Riobamba de la provincia de Chimborazo, dado que la población es finita se aplicó la fórmula de Aguilar [24] para calcular el tamaño de la muestra de un universo de 1646 Unidades Productivas Agropecuarias [25] (UPAs) detectadas mediante el levantamiento de información cartográfica.

$$
n_{? ?}=\frac{N Z ? ?}{(? ?-1)+? ?}
$$

Considerando un porcentaje de error del $5 \%$, el valor crítico $Z$ calculado de 1,96 , el nivel de precisión absoluta de 0,05 , los valores p y q de 0,5 , se obtuvo una muestra de 312 Unidades Productivas Agropecuarias, cada una de ellas lideradas por un jefe de familia.

El análisis de la presente investigación incluye un acercamiento con las directivas de las comunidades para fortalecer la gobernanza local [26], organización de asambleas con los productores para que las estrategias sean lo que esperan y para que interioricen las mismas y la aplicación de encuestas que permitan obtener datos base sobre las 3 dimensiones de sostenibilidad [27-29].

En la dimensión social se analizaron las variables: (i) edad, (ii) núcleo familiar, (iii) Nivel educativo y (iv) aporte e ingreso económico familiar (PEA), en la dimensión productiva se analizaron variables ecológicas, producción agrícola y producción pecuaria y, en la dimensión económica se analizaron los ingresos, gastos y cálculos de los indicadores económicos (TIR, VAN, B/C). 
Una vez obtenida la información de las UPAs, se construyeron estrategias utilizando la metodología de marco lógico [30] con la finalidad de que las mismas guarden coherencia y que al momento de ejecutarlas se pueda brindar un seguimiento y evaluación oportunas.

\section{Resultados}

\subsection{Análisis de las dimensiones de sostenibilidad}

\subsubsection{Dimensión social}

La edad promedio de los jefes de familia que se encuentran al frente de las UPAs es de 46 años, con una desviación estándar de 10,1, además, existe un 16\% de las UPAs que producen personas de la tercera edad. Siendo la mayor parte de las UPAs lideradas por personas jóvenes y adultas se puede incluir en la estrategia el fortalecimiento de capacidades en diferentes temáticas que aporten en la cadena productiva agropecuaria.

El 42\% de las familias está compuesta de 5 y 6 personas, mientras que el 38,2\% de familias cuentan con 3 y 4 personas, de ellas aproximadamente el 50\% aportan económicamente al hogar, siendo esos recursos no solo provenientes de la producción agrícola, sino también de la prestación de servicios como conductores, albañiles, guardianía y venta de mano de obra agropecuaria.

\subsubsection{Dimensión productiva}

\section{Variables ecológicas}

El suelo de la parroquia Quimiag tiene un gran potencial para actividades agropecuarias, en el $36,6 \%$ se produce pastos, $23,36 \%$ están cubiertas de páramo, mientras que el $7,37 \%$ son bosques naturales y el $4,58 \%$ corresponde a bosques plantados y apenas el $0,96 \%$ son áreas de conservación. Además, el $82 \%$ de los productores tienen acceso a un suelo de buena calidad debido al contenido de materia orgánica y el $18 \%$ mencionan que el suelo es regular ya que están ubicados en terrenos con pendientes pronunciadas, con gran presencia de rocas y que en el $21 \%$ de estos casos son suelos erosionados debido a la presencia de fuertes vientos o por el mal manejo de las prácticas de riego.

Asimismo, el 33\% de las UPAs no tienen plantas nativas y el $66,2 \%$ tienen plantas endémicas que ayudan a la conservación de suelo por equilibrar la microflora y microfauna que ayudan al control de plagas y enfermedades de los cultivos. Es decir, el suelo de Quimiag es apto para una producción sostenible tanto a nivel agrícola como pecuario.Con respecto al acceso de agua para riego, el 71\% de productores de Quimiag cuentan con 4 sistemas de riego, entre ellos: Quillán, Balcashí, Chambo y La Laguna, mientras que el $29 \%$ no dispone de regadío por lo que producen especies de secano. 
La parroquia de Quimiag cuenta con climas como el de alta montaña, clima semihúmedo, clima seco y clima nival [1].

\section{Producción agrícola}

La tenencia de tierra de los productores de la parroquia Quimiag es en promedio de 4,37 hectáreas, existiendo productores que poseen un máximo de 18 ha, así como también existen productores que poseen 1 ha para la producción agropecuaria familiar. Es decir, la tenencia de tierra es menor a la reportada por el INEC [2] en la provincia de Chimborazo que es de 6 ha, esto se debe principalmente a la parcelación de estas por motivos de herencias o reparto de tierras comunales.

Dentro de los principales rubros agrícolas cultivados en la parroquia Quimiag y que se muestran en la Tabla 1, tenemos al maíz que lo cultivan el $45,83 \%$ de las familias, seguido por el fréjol que lo cultivan el $39,10 \%$ y la papa que lo cultivan el $4,81 \%$ de las familias.

\section{Table 1}

Producción agrícola de Quimiag.

\begin{tabular}{lllll|l} 
Cultivos & Porcentaje & $\mathbf{N}^{\circ}$ de familias & Terreno (ha) & Cantidad $\mathbf{( K g . )}$ & $\begin{array}{l}\text { Costo promedio } \\
\mathbf{( \$ / K g )}\end{array}$ \\
\hline Maíz & 45,83 & 143 & 0,62 & 1620 & 1,5 \\
\hline Fréjol & 39,10 & 122 & 0,71 & 600 & 1,76 \\
\hline Papa & 4,81 & 15 & 0,54 & 10800 & 0,22 \\
\hline Haba & 3,85 & 12 & 0,58 & 470,16 & 1 \\
\hline Mora & 1,28 & 4 & 0,32 & 7360 & 0.95 \\
\hline Frutilla & 1,28 & 4 & 0,12 & 2760 & 0,95 \\
\hline Arveja & 1,28 & 4 & 0,12 & 1600 & 1 \\
\hline Zanahoria & 1,28 & 4 & 0,41 & 6150 & 0,25 \\
\hline Pasto & 1,28 & 4 & 0,94 & 61,100 & 0,1
\end{tabular}

Fuente: Arévalo (2020).

La producción agrícola se destina principalmente para el autoconsumo, siendo los principales rubros consumidos las papas (25\%) y las habas (17\%). Asimismo, productos como el maíz (29\%), fréjol (19\%), zanahoria (20\%), papas (7\%) y habas (4\%) generan excedentes que se comercializan en los principales mercados de la ciudad de Riobamba.

La percepción de los productores agrícolas con respecto a las limitantes de producción es: Incidencia de plagas y enfermedades (49\%); presencia de plagas y desconocimiento (26\%); además, un $6 \%$ menciona que el principal problema son las plagas y la semilla de mala calidad; plagas y comercialización (6\%) y el $26 \%$ dice que las plagas y la falta de crédito son las limitantes para una óptima producción agrícola. Como se puede apreciar el factor plagas es lo que más percibe los productores como problemas presentes en los procesos productivos. Asimismo, dentro de los riesgos naturales tal como se muestra en la Figura 1, mencionan que el cambio climático ha 
afectado al 32\% de las UPAs, debido a que ya no es marcado la temporada de lluvia en la que sembraban, también el $25 \%$ mencionan que la sequía menora la producción de maíz y de fréjol.

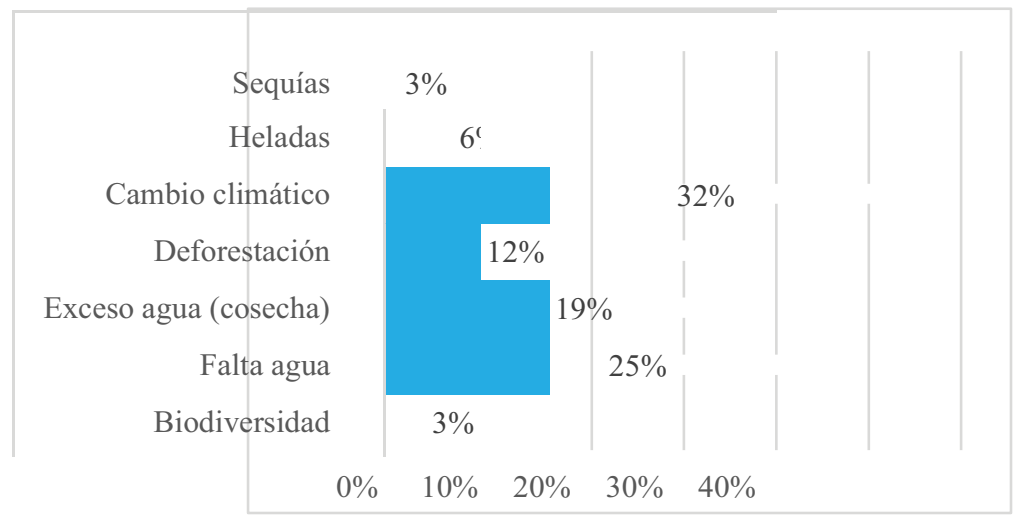

Figure 1

Factores ambientales que indicen en los procesos productivos de Quimiag.

\section{Producción pecuaria}

Tal como se presenta en la Tabla 2, en la parroquia Quimiag, los rubros pecuarios más importantes son; ganado de leche (91\%), cuyes (76\%), porcinos (31\%), y un $4 \%$ se dedica a la producción de aves; las aves y cuyes se destinan principalmente para el autoconsumo, considerándolos como alimento de festividades o para eventos comunales especiales.

\section{Table 2}

Distribución porcentual de las especies explotadas en las unidades de producción sujetas a estudio.

\begin{tabular}{l|ll} 
Detalle & Frecuencia & Relativa (\%) \\
& Absoluta (\#) & 76 \\
\hline Cuyes & 78 & 19 \\
\hline Ovinos & 19 & 4 \\
\hline Aves & 4 & 31 \\
\hline Porcinos & 32 & 91 \\
\hline Bovinos de leche & 93 & 13 \\
\hline Bovinos de carne & 13 &
\end{tabular}

Fuente: Arévalo (2020).

\subsubsection{Dimensión económica}

El análisis financiero se elaboró de acuerdo con la información de los productores de la parroquia Quimiag, en la misma que se consideró la construcción de corrales, insumos 
de ordeño, materiales para la producción adecuada de ganado bovino, promedios de producción principalmente de la leche, comederos dando como resultado los datos mostrados en la Tabla 3, en los que se mencionan las variables de TIR, VAN y Relación Beneficio- Costo.

\section{Table 3}

Variables económicas de la producción agropecuaria de la parroquia Quimiag.

\begin{tabular}{|c|c|c|c|}
\hline Condiciones & TIR (\%) & VAN (USD) & $\mathrm{B} / \mathrm{C}$ (USD) \\
\hline Normal & 9 & 7734,05 & 1,05 \\
\hline Sube Egresos $5 \%$ & 0 & $-49,02$ & 1,00 \\
\hline Baja Ingresos $5 \%$ & 0 & $-435,72$ & 1,00 \\
\hline $\begin{array}{l}\text { Ingresos baja y } \\
\text { Egresos. Sube 5\% }\end{array}$ & -8 & $-8218,79$ & 0,95 \\
\hline
\end{tabular}

Fuente: Arévalo (2020).

Como se puede apreciar en condiciones normales se obtiene un Valor Agregado Neto de 7734.05 USD, pero si existe una subida en egresos o bajan los ingresos en un $5 \%$ mientras tanto si sube los egresos o a su vez baja los ingresos en un $5 \%$ no existiría rentabilidad en la producción agropecuaria. Asimismo, la Tasa Interna de Retorno tiene un comportamiento similar, siendo de $9 \%$ en condiciones normales, pero llegando a ser de $0 \%$ o - $8 \%$ en el caso de que los ingresos bajen y los egresos suban en un $5 \%$. La relación Beneficio Costo es de 1,05 USD en condiciones normales, es decir existiría una ganancia de 0,05 por cada dólar de inversión en las UPAs [31].

\subsection{Estrategias para el fortalecimiento de las unidades de produc- ción con enfoque de marco lógico}

El enfoque de Marco Lógico sirvió como herramienta para elaborar las estrategias de sostenibilidad para el fortalecimiento de los sistemas agropecuarios de la parroquia Quimiag, las mismas que fueron realizadas de manera participativa con los productores mediante asambleas comunitarias en las que se usaron varias metodologías que nos permitieron conocer los problemas, y sobre estos se pudo plantear objetivos, además se pudo identificar los actores que participan en la parroquia y que apoyan en los procesos productivos, estableciendo varias alternativas de acción y analizando las mismas.

\subsubsection{Marco lógico}

\section{Propuesta técnica}

\section{Descripción de la propuesta}

Los beneficiarios directos serán los productores de las 24 comunidades de la parroquia Quimiag, pudiendo llegarse de manera indirecta a apoyar a las 31 comunidades existentes. 


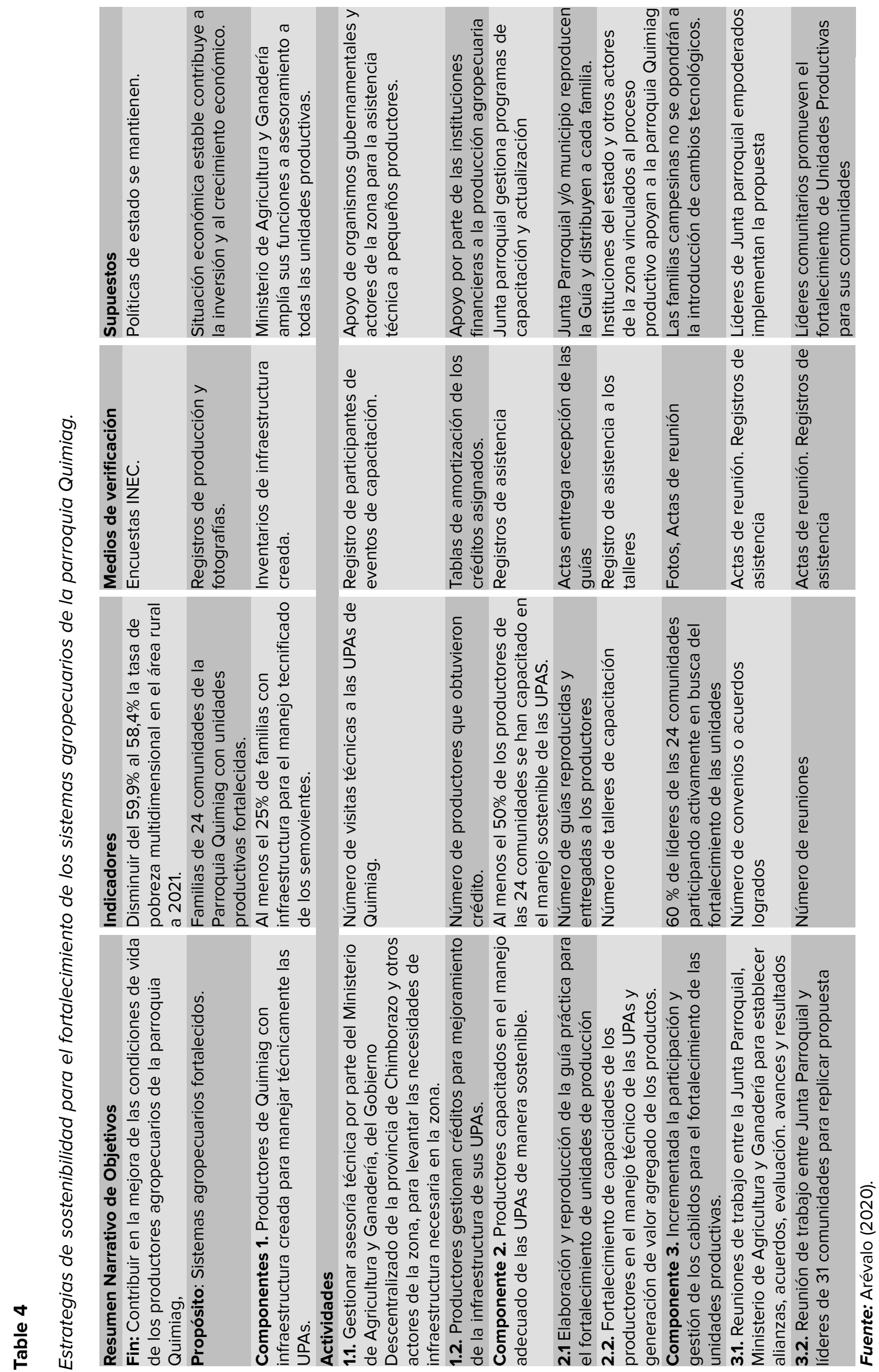




\section{Actividades de la propuesta}

Las actividades se basan en la Tabla 4, para cumplimiento de los componentes planteados, los mismos que ayudarán a mejorar la calidad de vida de los productores agropecuarios de la parroquia Quimiag y por ende de sus familias. Entre las actividades tenemos:

\section{Fortalecimiento de capacidades y generación de vínculos}

En el componente 1 y 2 de la propuesta es necesario fortalecer las capacidades para mejorar la infraestructura de los sistemas de producción de la parroquia Quimiag, pero también en el manejo de los rubros existentes y la diversificación de la producción. Además, se propone generar un espacio de formación de líderes y lideresas comunitarias como estrategia de multiplicar el conocimiento no solo en las 24 comunidades participantes sino llegar a las 31 comunidades existentes en Quimiag. En el proceso de fortalecimiento de capacidades se tomará en cuenta que 18 comunidades son es su mayor parte de habla hispana, mientras que en los 13 restante predominan los Kichwa hablantes [1]. Para cumplimiento de esta estrategia se deben generar alianzas con instituciones públicas y privadas, sobre todo con los actores que apoyan en la zona para que exista asesoría técnica que beneficien a los productores del total de comunidades de Quimiag.

Asimismo, se deberán generar acuerdos con entidades financieras que permitan a los productores tener acceso a créditos para fortalecimiento de sus UPAs.

\section{Mejoramiento productivo}

Debido a la topografía de la zona y pensando en la sostenibilidad de los sistemas se debe incluir prácticas de conservación de suelos, asimismo, ya que la parroquia cuenta con un suelo apto para la producción agropecuaria se deberá diversificar la producción.

El agua es un recurso con el que si bien es cierto el $71 \%$ de los productores cuentan, de ellos, el $90 \%$ tiene sistema de riego por aspersión, el $2 \%$ tiene un sistema por goteo y un $8 \%$ realiza el riego por gravedad; el $29 \%$ restante han tenido pérdidas de su producción por no contar con este factor que es limitante de su producción. Con este antecedente se considera que se debe trabajar con las juntas de regantes en el fortalecimiento de capacidades sobre la importancia del uso y manejo de este recurso.

El mejoramiento de la producción agrícola debe basarse en conocimientos del manejo del recurso suelo, conocimiento del ciclo productivo de los principales rubros, así como también de otros en los que se puede potenciar la producción. Se debe también mejorar el conocimiento de la sanidad de las plantas y el uso y manejo de fertilizantes y plaguicidas, incluyendo temas de producción de insumos ecológicos que sean más amigable con el medio ambiente.

Para mejorar la producción pecuaria se requiere del conocimiento de los productores sobre el manejo productivo y reproductivo de los principales rubros pecuarios, así como 

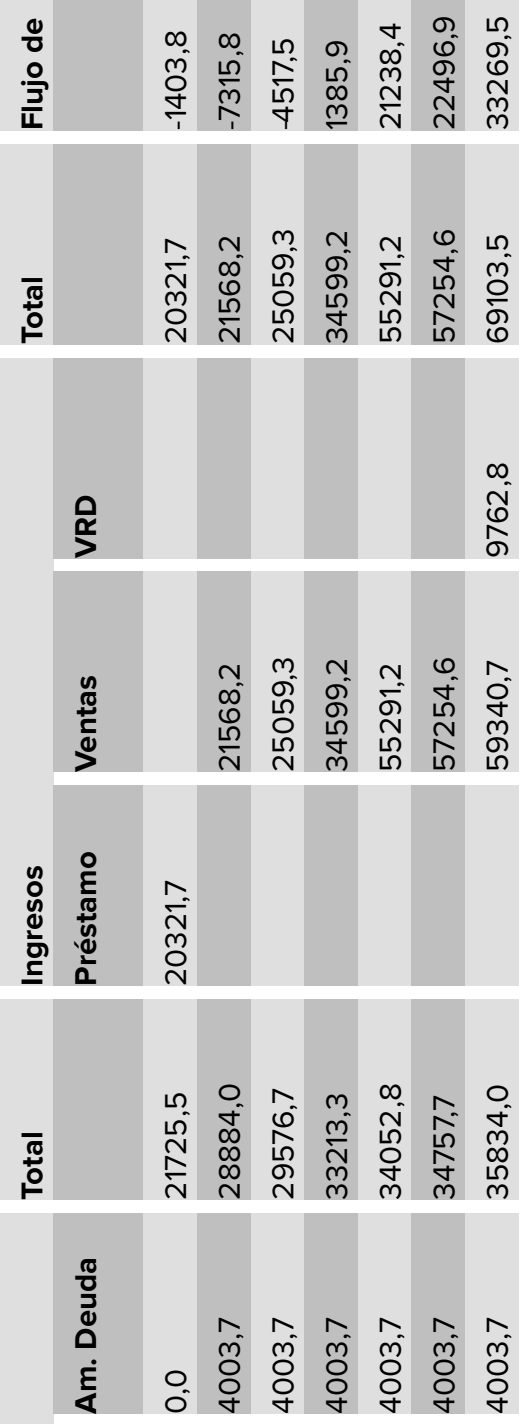

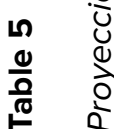

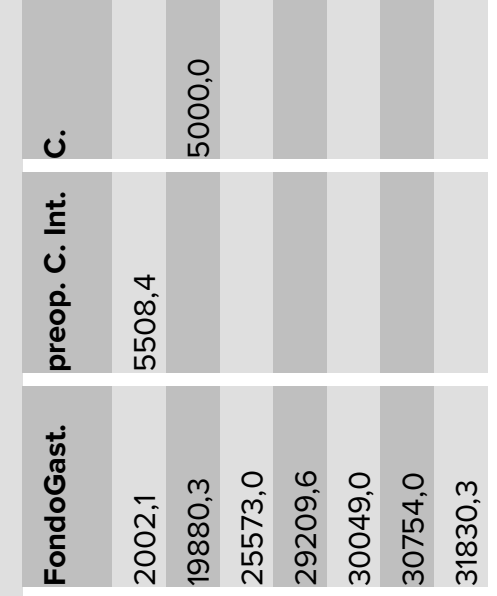

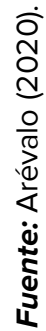


también su manejo sanitario. Es importante además se debe trabajar en el mejoramiento de los pastos que son la base de la alimentación animal.

\subsubsection{Análisis económico}

El cálculo de los indicadores económicos de la propuesta se basa en la tenencia de tierra promedio de los productores que es de 4,37 ha y en sus sistemas de producción en los principales rubros agrícolas que son el maíz, fréjol y papas, y en el caso de la producción pecuaria basado en el ganado lechero, cuyes y cerdos, con esos datos se realizó la proyección de Ingresos y Egresos que se puede evidenciar en la Tabla 5.

Con esa información, los indicadores económicos nos indican que existe una rentabilidad con un VAN de 48654,43 USD, una TIR de 52\% y una relación Beneficio costo de 1,26 , estos en condiciones normales, pero es importante recalcar que sus valores son apreciables aun cuando suben los egreso y bajan los ingresos en un $5 \%$ como se lo indica en la Tabla 6.

\section{Table 6}

Indicadores económicos de la propuesta.

\begin{tabular}{l|l|l|l}
\hline Condiciones & TIR (\%) & VAN (USD) & B/C (USD) \\
\hline Normal & 52 & 48654,43 & 1,26 \\
\hline Sube Egresos 5 \% & 37 & 39317,39 & 1,20 \\
\hline Baja Ingresos 5 \% & 36 & 36884,67 & 1,20 \\
\hline Ing baja Eg. Sube 5 \% & 24 & 27547,63 & 1,14
\end{tabular}

Fuente: Arévalo (2020).

\section{Conclusiones}

- Las Unidades Productivas Agropecuarias (UPAs) de la parroquia Quimiag, están lideradas por los jefes de hogar cuya edad promedio es de 46 años, siendo el promedio de tenencia de tierra de los productores de Quimiag de 4,37 ha y cuentan con 4 sistemas de Riego que sirven para la producción agropecuaria que se basa principalmente en los rubros agrícolas como maíz, fréjol y papa; y en los rubros pecuarios como: bovinos de leche (91\%), Cuyes (76\%) y porcinos (31\%).

- Los indicadores económicos de los sistemas productivos ex-ante en un escenario normal tienen un VAN de 7734,05 USD, una TIR es de 9\% y una relación Beneficio-Costo de 1,05 , mismos que son susceptibles a la subida de egresos o baja de ingresos del $5 \%$, los cuales ya no generarían rentabilidad, mientras que con la generación de estrategias sostenibles como son: Mejorar el manejo técnico de los cultivos mediante el fortalecimiento de capacidades de los productores, la tecnificación de la producción, la gestión de créditos productivos y la generación de alianzas estratégicas con instituciones del estado y empresas 
privadas, existe una mejora de los indicadores económicos, obteniéndose así un VAN $=48654,43$, una TIR del 52\% y una relación Beneficio-Costo de 1,26.

\section{References}

[1] Guapi R, Masaquiza D, Curbelo L. Caracterización de sistemas productivos lecheros en condiciones de montaña, Parroquia Químiag, Provincia Chimborazo, Ecuador. Revista de producción animal. 2017;2:1424.

[2] INEC 2018. Boletín técnico N 01-2018-ESPAC Encuesta de superficie y producción agropecuaria, continua. Ecuador; 2016.

[3] Aldunate E, Córdoba J. Formulación de programas con la metodología de marco lógico. CEPAL; 2011.

[4] Petersen J, Alert F, Vásquez $Y$ et al. Ensayos para un modelo de desarrollo sostenible: un cambio estructural. Santiago de Chile: Instituto de Ciencias Alejandro Lipschutz; 2017.

[5] Bielschowsky R, Torres M, Cepal N. Desarrollo e igualdad: El pensamiento de la CEPAL en su séptimo decenio. Textos seleccionados del período 2008-2018. CEPAL; 2018.

[6] Trapero J. Desarrollo sostenible y bienestar social: Una referencia a la comunidad de Galicia. Revista Galega de Economía. 2004;13:1-2.

[7] Munasinghe M. Economía ambiental y desarrollo sostenible. El Banco Mundial; 1993.

[8] Trapero J. Desarrollo sostenible y bienestar social: Una referencia a la comunidad de Galicia. Revista Galega de Economía. 2004;13(1-2).

[9] Higón F. Tamarrit. Desarrollo económico y sostenible. Valecia: Madrid; 1900.

[10] Barreras I. El desarrollo tecnológico y la innovación como ente principal de competitividad en las empresas del sector agropecuario en el municipio de Angostura, Sinaloa. Revista Mexicana de Agronegocios. 2018;42:867-877.

[11] Acevedo A, Linares M. El enfoque y rol del ingeniero industrial para la gestión y decisión en el mundo de las organizaciones. Ind. Data. 2013;3:9-14.

[12] Daly H. Desarrollo sustentable definiciones, principios y políticas. 2008.

[13] Cabrera C, Espinosa B. Desafíos para la educación en el Ecuador: Calidad y equidad. Quito: FlacsoSede; 2008.

[14] Rico M. Género, medio ambiente y sustentabilidad del desarrollo. Santiago de Chile: Naciones Unidas; 1998.

[15] Tonolli A, Greco S, Sarandón S. Algunos aspectos emergentes y de importancia para la construcción del enfoque agroecológico. Revista de la Facultad de Ciencias Agrarias UNCuyo. 2019;51:205-212.

[16] Anyaegbunam C, Mefalopulos P, Moetsabi T. Evaluación Rural Participativa Comunicación. Roma: 2004

[17] Hidalgo A, et al. Orto y ocaso del buen vivir en la planificación nacional del desarrollo en Ecuador (2007-2021). Quito; 2018.

[18] Falconí F. Economía y desarrollo sostenible matrimonio feliz o divorcio anunciado? Quito: Sede Académica de Ecuador; 2002.

[19] Artaraz M. Teoría de las tres dimensiones de desarrollo sostenible. Revista Ecosistemas. 2002;11:2.

[20] Toledo A. Programa de las Naciones Unidas para el Medio Ambiente-Oficina Regional para América Latina y el Caribe. Economía de la biodiversidad; 1998.

[21] Guamán S. El Sector agrícola-ganadero y su aporte al desarrollo local de la parroquia químiag, provincia de chimborazo, durante el período 2014-2015 [Bachelor's thesis]. Riobamba: Universidad Nacional de Chimborazo; 2016.

[22] Fernández N, Schejtman, L. Planificación de políticas, programas y proyectos sociales. Buenos Aires: UNICEF; 2012.

[23] BID Banco internacional de Desarrollo. El Marco Lógico para el Diseño de Proyectos. 2005.

[24] Dennis R. Cómo estimar el tamaño de la muestra en investigaciones con humanos. Acta Médica Colombiana. 1989;14:92-9.

[25] Lugo L. Análisis del servicio de asistencia técnica ejecutado por la unidad municipal de asistencia técnica agropecuaria-Umata (periodo 1.998-2.007) en el municipio de Florencia, Caquetá [Tesis de posgrado]. Bogotá: Pontificia Universidad Javeriana; 2009.

[26] Beas D. Gobernanza para el desarrollo local. Documento de trabajo N. 2011;6.

[27] FAO. Desarrollo agropecuario de la dependencia al protagonismo del agricultor. Chile: FAO; 1995.

[28] Devé F. Género y sistemas de producción campesinos. 2005. 
[29] Gavín B. Propuesta de un plan de desarrollo de turísmo sostenible para la parroquia de Lícto, Cantón Riobamba. 2013. Disponible en: http://dspace.espoch.edu.ec/bitstream/123456789/2767/1/ 23T0362\{\%\}20\{\%\}20GAVIN\{\%\}20BRENDA.pdf

[30] Coneval. Guía para la elaboración de la matriz de indicadores para resultados. México; 2013.

[31] Arévalo M. Propuesta para la sostenibilidad de las unidades de producción agropecuarias campesinas de la parroquia Quimiag [Tesis de posgrado]. Riobamba: ESPOCH; 2020. 